\title{
Huachansu suppresses human bladder cancer cell growth through the Fas/Fasl and TNF- alpha/TNFR1 pathway in vitro and in vivo
}

Tao Yang ${ }^{1 \dagger}$, Runlin Shi ${ }^{1 \dagger}$, Lei Chang ${ }^{2}$, Kun Tang ${ }^{1}$, Ke Chen ${ }^{1}$, Gan Yu ${ }^{1}$, Yuanfeng Tian ${ }^{1}$, Yonglian Guo ${ }^{2}$, Wei He , Xiaodong Song ${ }^{1}$, Hua $\mathrm{Xu}^{1 *}$ and Zhangqun $\mathrm{Ye}^{1 *}$

\begin{abstract}
Background: Huachansu (HCS), a class of toxic steroids extracted from toad venom, which has been shown to be a valuable anticancer drug in many kinds of cancers. However, the effect of HCS on bladder cancer has not been elucidated. In this study, we focused on the antitumor activities and related mechanisms of HCS on bladder cancer in vitro and in vivo.

Methods: Cell viability of T24, EJ, RT-4, SV-HUC-1 cells after HCS treatment was measured by MTS, whereas the changes of cell morphology were observed by transmission electron microscopy. The early apoptosis induced by HCS was evaluated by flow cytometry, and the expression level of apoptosis-related molecules (Bax, BCl-2, XIAP, PARP, cleaved-Caspases 3, 8, 9) was detected using Western blot. We then evaluated the impact of HCS on the expression of Fas/Fasl, TNF- alpha/TNFR1, and the activation of NF-Kappa B pathway, and furthermore the effect of these pathways in HCS induced-apoptosis were also detected. At last, xenograft tumor in nude mice was used to further investigate the antitumor effect of HCS in vivo.

Results: Our results showed that HCS could efficiently inhibit proliferation and induce apoptosis in human bladder cancer cell lines. The expression of Fas, Fasl, TNF- alpha were all elevated at both mRNA and protein level after HCS treatment. Furthermore, down regulation of TNF- alpha, TNFR1, Fas or inhibition of Fas/Fasl interaction decreased the relative number of death cells induced by HCS. In vivo, HCS treatment significantly suppressed tumor growth and induced apoptosis in xenografts tumor in nude mice.
\end{abstract}

Conclusions: HCS could efficiently inhibit proliferation and induce apoptosis in human bladder cancer cells in vitro and in vivo, which is largely mediated by Fas/Fasl and TNF- alpha/TNFR1 pathway.

Keywords: Huachansu, Bladder cancer, Apoptosis, TNF- alpha/TNFR1, Fas/Fasl

\section{Background}

Bladder cancer is a major public health problem worldwide. More than $90 \%$ of the bladder cancers are urothelial cell carcinomas (UCC) and approximately $70 \%$ of bladder tumor present as non-muscle-invasive bladder cancer (NMIBC) [1,2]. The standard treatment for patients with superficial bladder cancer is transurethral resection (TUR) of tumors. However, nearly $60 \%$ to $70 \%$

\footnotetext{
*Correspondence: xuhuawhu@163.com; zhangqun_ye@163.com ${ }^{\dagger}$ Equal contributors

'Department and Institute of Urology, Tongji Hospital, Tongji Medical College, Huazhong University of Science and Technology, Wuhan 430030, China

Full list of author information is available at the end of the article
}

of these tumors will recur, and $25 \%$ will progress into a higher stage or grade $[3,4]$. Although many chemical agents have shown some evidence of activity against tumor recurrence, their toxicity and incomplete efficacy have limited their use as common chemotherapy agents [5]. These factors highlight the urgent of novel adjuvant agents. Natural products, including those from plants and microorganisms, provide much potential for anticancer drug discovery [6,7].

Chansu, the dried toad venom or the dried secretion from the skin glands of Bufo bufo gargarizans Cantor or B.melanotictus Schneider has long been used for cancer treatment in China and other Asian countries, such as 
the treatment for liver and pancreatic cancer [8-10]. Huachansu (HCS) is an injectable form of chansu, the extract contains several biologically active substances, primarily indole alkaloids (bufotenine, bufotenidine, and cinobufotenine) and steroidal cardiac glycosides (bufalin, resibufogenin, cinobufagin, cinobufotalin, marinobufagin, and bufotalin). Recent studies showed that the antitumor activity of HCS can be attributed mainly to the cardiac glycosides it contains, including bufalin, resibufogenin, and cinobufagin. High-pressure liquid chromatography (HPLC) analysis determined the relative concentrations of the components. Bufalin was present at the highest concentration, followed by moderate levels of cinobufagin [11]. Chansu extractions including Bufalin and cinobufagin induce apoptosis and inhibit proliferation of many cancer cells through different pathways, Bufalin induces apoptosis by activation of activator protein-1 (AP-1), the c-JunN-terminal protein kinase, Rac1, mitogen-activated proteinkinase $[9,12]$ in human leukemia U937 cells, as well as by inhibition of phosphatidylinositol 3-kinase (PI3K)/Akt signaling in human gastric cancer cells [13]. Bufalin and cinobufagin induce apoptosis of human prostate cancer cells partialy with Fas stimulation, Bax translocation, cytochrome c release and caspase activation [14]. However, the effect of HCS on bladder cancer has not been elucidated. So in this study, we investigated the anticancer effects and related molecular mechanisms of HCS on bladder cancer in vitro and in vivo.

\section{Methods}

\section{Cell culture and reagents}

Bladder cancer cell lines T24 and EJ were obtained from the Institute of Biochemistry and Cell Biology, Shanghai Institutes for Biological Sciences, Chinese Academy of Sciences (Shanghai, China), and maintained in RPMI1640 medium. SV-HUC-1 and RT-4 were also purchased from this place and maintained in DMEM/F-12 and McCoy's 5A medium respectively. Cells were cultured added with $10 \%$ fetal bovine serum (FBS) in a humidified atmosphere of $5 \% \mathrm{CO}_{2}$ maintained at $37^{\circ} \mathrm{C}$. $\mathrm{HCS}$ was obtained from Anhui Jinchan Biochemistry Company Ltd., in Huaibei, China (Chinese FDA (Z34020274)), PDTC was purchased from Sigma-Aldrich and ZB4 was bought from GeneTex.

\section{Cell viability assay}

Briefly, T24, EJ, RT-4 and SV-HUC- 1 cells $\left(3 \times 10^{3}\right.$ per well) were plated in 96-well plates and incubated with various dilutions of standard $\operatorname{HCS}(1: 400,1: 200,1: 100$, 1:50) for 24, 48 and $72 \mathrm{~h}$. Cell viability was then detected using CellTiter $96^{\circ} \mathrm{AQueous} \mathrm{Non-Radioactive} \mathrm{Cell} \mathrm{Pro-}$ liferation Assay (MTS) (Promega, USA). Three independent experiments with triplicate were carried out.

\section{Transmission electron microscopy}

T24 cells were pretreated with the indicated dilution of HCS(1:100) for $24 \mathrm{~h}$ and $48 \mathrm{~h}$, respectively. The treated cells were then collected and fixed with $2.5 \%$ glutaraldehyde. Ultrastructure of the cells was examined on a transmission electron microscopy (Tecnai, Netherlands) at $2500 \times$ magnification.

\section{Flow cytometry}

Cellular apoptosis was determined by annexin V-FITC/ PI staining using flow cytometry. T24 and EJ cells were pretreated with different dilutions of HCS for $24 \mathrm{~h}$. After that, cells $\left(1 \times 10^{6}\right)$ were collected, centrifuged and washed with phosphate buffered saline(PBS) for two times. $300 \mu \mathrm{L}$ Binding buffer was then added to each tube and cells were re-suspended. The supernatant cells were incubated with $5 \mu \mathrm{L}$ of annexin V-FITC and $5 \mu \mathrm{L}$ of PI for $15 \mathrm{~min}$ at room temperature in the dark. Then, the apoptotic analyses were done by flow cytometry within one hour.

\section{RNA extraction and quantitative real-time RT-PCR}

Total RNA was extracted with the TRIzol reagent (Invitrogen Life Technologies). RNA yield and quality were determined with a Nano Drop 1000 Spectrophotometer. For cDNA synthesis, $1 \mu \mathrm{l}$ of total RNA was reversetranscribed using Toyobo RT reagent Kit (Perfect Real Time) according to the manufacturer's instructions. Quantitative real-time RT-PCR was carried out using using SYBR Premix Ex Taq on MX3000 instrument. The PCR amplification program consisted of an initial polymerase activation at $95^{\circ} \mathrm{C}$ for $10 \mathrm{~min}$, followed by 40 cycles at $95^{\circ} \mathrm{C}$ for $5 \mathrm{~s}, 60^{\circ} \mathrm{C}$ for $30 \mathrm{~s}$ and $72^{\circ} \mathrm{C}$ for $30 \mathrm{~s}$ for those for genes. The results were normalized with housekeeping gene Beta-actin. Sequences of primers are listed in Table 1.

\section{Protein extraction and Western blot analysis}

Cells after treated with HCS were harvested and lysed with lysis buffer (50 mM Tris (pH7.4), $50 \mathrm{mM} \mathrm{NaCl}$, $1 \mathrm{mM}$ EDTA, $1 \%$ Triton X-100, and 10\% glycerol). All solutions contains protease inhibitor cocktail (Sigma) and mixture of phosphatase inhibitors $(10 \mathrm{mM} \mathrm{NaF}$, $1 \mathrm{mM}$ sodium pyrophosphate, $1 \mathrm{mM}$ sodium orthovanadate, and $0.1 \mathrm{mM} \beta$ - glycerophosphate), and the protein extraction was processed according to the manufacturer's instructions. Protein concentration was measured using a BCA Protein Assay Kit (Beyotime, Wuhan, China) according to the manufacturer's instructions. For Western blot analysis, an equal amounts of $50 \mu \mathrm{g}$ protein were subjected to electrophoresis on SDS-polyacrylamide gels and transferred onto polyvinylidene fluoride (PVDF) transfer membranes by western blotting. Blots were probed with the following antibodies Bax, Bcl-2, XIAP, 
Table 1 Primer sequences for RT-PCR

\begin{tabular}{llll}
\hline Target & Upstream primer & Downstream primer & Accession number \\
\hline Fas & TCTGGTTCTACGTCTGTTGC & CTGTGCAGTCCCTAGCTTTCC & NM_152872 \\
Fasl & AACTCAAGGTCCATGCCTCTG & GGTGAGTTGAGGAGCTACAGACA & NM_000639 \\
TNF-a & TGTAGCCCATGTTGTAGCAAA & CAAAGTAGACCTGCCCAGACT & NM_000594 \\
TNFR1 & TCCTTCACCGCTTCAGAAAA & GGGATAAAAGGCAAAGACCAA & NM_001065 \\
$\beta$-actin & TCCTGACCCTGAAGTACCCCATTG & GGAACCGCTCATTGCCGATAGT & NM_001101 \\
\hline
\end{tabular}

cleaved Caspase-3,-8,-9, cleaved PARP, p65, p-p65, iкB- $\alpha$ (Cell Signaling Technology, USA), Fas, Fasl, (epitomics, USA) and TNFR1 (Proteintech group,USA ) overnight at $4^{\circ} \mathrm{C}$. Then followed by secondary antibody-conjugated horseradish peroxidase (HRP) and detected by ECL solution .

\section{ELISA assay for TNF- $a$ secretion}

After cells were treated with HCS for $4 \mathrm{~h}$ and $8 \mathrm{~h}$, the level of TNF- $\alpha$ protein accumulating in the medium were determined using an TNF- $\alpha$ ELISA kit (ExCell Biology, Shanghai, China). The assay was performed in duplicate according to the manufacturer's recommendations.

\section{SiRNA trasfection}

siRNA targeted against Fas (5'- GUGCAAGUGCAAACC AGACTT -3'), TNFR1 (5'- CAAAGGAACCUACUUGU ACUU -3'), TNF-a (5'- GUGCUGGCAACCACUAA GA -3';) or control siRNA (5'-CCCCUUUUAAAA GGGGCCC-3') was transfected into T24 and EJ cells using Lipofectamine RNAiMAX (Invitrogen) to a final concentration of $50 \mathrm{pmol} / \mathrm{ml}$ according to the manufacturer's recommendations. 48 hours after transfection, knockdown was assessed by PCR from a parallel transfection.

\section{Bladder cancer xenograft model and in vivo therapy with HCS}

All animal procedures were carried out with the approval of the Animal Ethics Committee of the Huazhong University of Science and Technology. 4-week old female athymic nude mice (BALB/c-nu/nu mice) were inoculated subcutaneously with $5 \times 10^{6}$ T24 cells. After 7 days, $98 \%$ of mice grew visible tumors. The mice were randomized and assigned to the control group or the experimental group. Mice in the control group were percutaneously injected with $0.4 \mathrm{ml}$ saline daily, and mice in the experimental group were percutaneously injected with $0.4 \mathrm{ml} \operatorname{HCS}(1: 2,1: 1)$ into the tumor every day. The tumors were measured twice a week with microcalipers, and the tumor volumes were calculated using the following equation: Tumor volume $\left(\mathrm{mm}^{3}\right)=1 / 2 \times$ (tumor length) $\times$ (tumor width $)^{2}$. And the body weight of the mice was recorded twice a week. At the end of experiment, tumors were excised, weighed, and then each tumors were fixed in $4 \%$ of paraformaldehyde for further analyses.

\section{In situ apoptosis detection by TUNEL staining}

After desired treatment, the paraffin-embedded sections of samples were studied by terminal deoxynucleotidyl transferase-mediated dUTP nick-end-labeling (TUNEL) assay. Staining was carried out according to the protocol provided by the supplier. Apoptosis was evaluated by counting the positive cells as well as the total number of cells at 10 arbitrarily selected fields at $400 \times$ magnification in a blinded manner.

\section{Immunohistochemistry staining}

Immunohistochemistry (IHC) was conducted as previously described [15]. Tissues were deparaffinized, rehydrated, and incubated at room temperature in $0.3 \% \mathrm{H}_{2} \mathrm{O}_{2}$ to block endogenous peroxidase and in blocking solution for nonspecific binding. Primary antibody were applied to sections overnight at $4^{\circ} \mathrm{C}$. Afterwards, tissues were incubated with anti-mouse HRP conjugated (Abcam, USA) secondary antibody for $1 \mathrm{~h}$ at room temperature. Then enzyme development was performed with DAB/ $\mathrm{H}_{2} \mathrm{O}_{2}$ complex for $10 \mathrm{~min}$ at room temperature and in the absence of light which provides a brownish precipitation. The primary antibodies specific for Fas, Fasl (epitomics, USA) ,TNF- $\alpha$ and TNFR1(Proteintech group, USA) were used at working dilution 1:50, 1:100, 1:50 and 1:200 respectively. Stained (brown) cells were quantified as number of positive cells. To evaluate the intensity of antigen immunoreactivity we examined the percent of positive staining urothelial cells in 10 fields at random per rat per antibody under $400 \times$ magnification.

\section{Statistical analysis}

Statistical analysis was performed using the software of Statistical Package for the Social Sciences Version 16 for Windows. Data from 3 to 5 independent experiments were calculated as means and standard deviations. Comparisons of results between treated and control groups were made by the Student $t$ tests. A P-value of less than 0.05 was considered significant. 


\section{Results}

HCS inhibits the viability of human bladder cancer cells After cells were treated with various dilutions of HCS for 24,48 and $72 \mathrm{~h}$, cell viability of T24 and EJ, measured by the MTS assay, decreased significantly in a dose- and time-dependent manner (Figure 1A). Meanwhile, HCS showed little inhibition effect on less malignant RT-4 cells and immortalized SV-HUC-1 cells. This phenomenon indicated that HCS may have less damage on normal bladder tissue.

\section{Morphologic change induced by HCS}

To get some detailed morphologic changes, we used transmission electron microscopy. As shown in Figure 1B, The normal cells (normal saline) showed untreated cells with intact nuclear membrane, huge and circular nuclei, more chromatin, abundant mitochondria and endoplasmic reticulum with good morphology. Compared with the control, the cells treated with HCS for $24 \mathrm{~h}$ exhibited that the chromatin accumulation inside the nuclear membrane lumped, a large number of autophagocytic vacuoles formed, and the mitochondria were damaged. After cells were incubated for $48 \mathrm{~h}$, the cells became smaller, organelles were destroyed, partial nuclear membranes were disrupted and nuclei broke up.

\section{Apoptosis effect of HCS on bladder cancer cells}

The apoptotic effect of HCS on bladder cancer cells was detected through Annexin V-FITC/PI double staining assay. Results demonstrated that HCS had an effect on increasing apoptosis. With Annexin V staining, early apoptosis was clearly detectable in the two bladder cancer cells treated with HCS. Compared to the control group, the cell apoptotic rates were significantly increased in a dose-dependent manner (Figure 1C).

\section{The effect of HCS on apoptosis-related molecules}

To investigate the mechanism behind HCS-induced apoptosis, we detected the level of several apoptosisrelated molecules by western blot. As is shown in Figure 2, the results revealed that the levels of the active (cleaved) forms of Caspase-3,-8,-9 and cleaved PARP were increased in a dose-dependent manner in HCS-treated T24 and EJ cells. We next determined whether HCS-induced apoptosis in bladder cancer cells is also associated with the modulation of the inhibitors of apoptosis proteins (IAPs) and Bcl-2 family proteins. Western blotting results indicated that HCS treatment decreased the expression of Bcl-2 and increased the expression of Bax. The expression of XIAP was also significantly decreased in both two cell lines after HCS treatment.

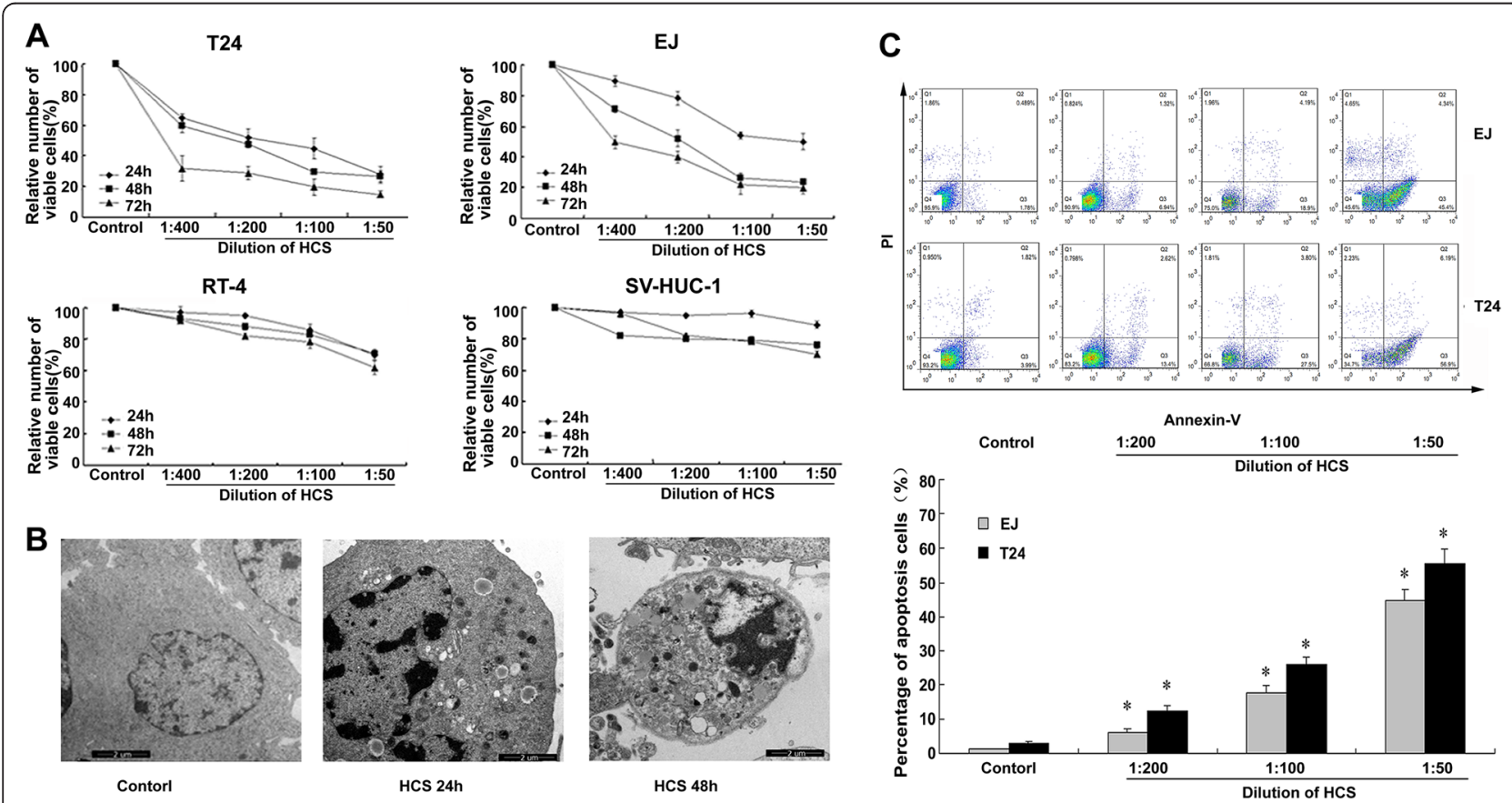

Figure 1 HCS prohibited bladder cancer cells growth and induced apoptosis of T24 and EJ cells. (A) Effect of HCS on proliferation of T24, EJ, RT-4, and SV-HUC-1 cells. Values are given as a percentage of untreated control cells. The data are presented as the average for triplet results from a representative experiment; bars, SD. (B) The ultrastructural morphologic changes of the T24 cells treated with HCS (1:100) under the transmission electron microscope. (C) The apoptotic fraction of cells detected by annexin $V$ staining ( $x$-axis)/propidium iodide staining ( $y$-axis) after HCS treatments and the percentages showing the annexin V-positive/PI-negative fraction. Columns are expressed as mean \pm SD of 3 independent experiments. ${ }^{*}, p<0.05$ for HCS vs. control. 


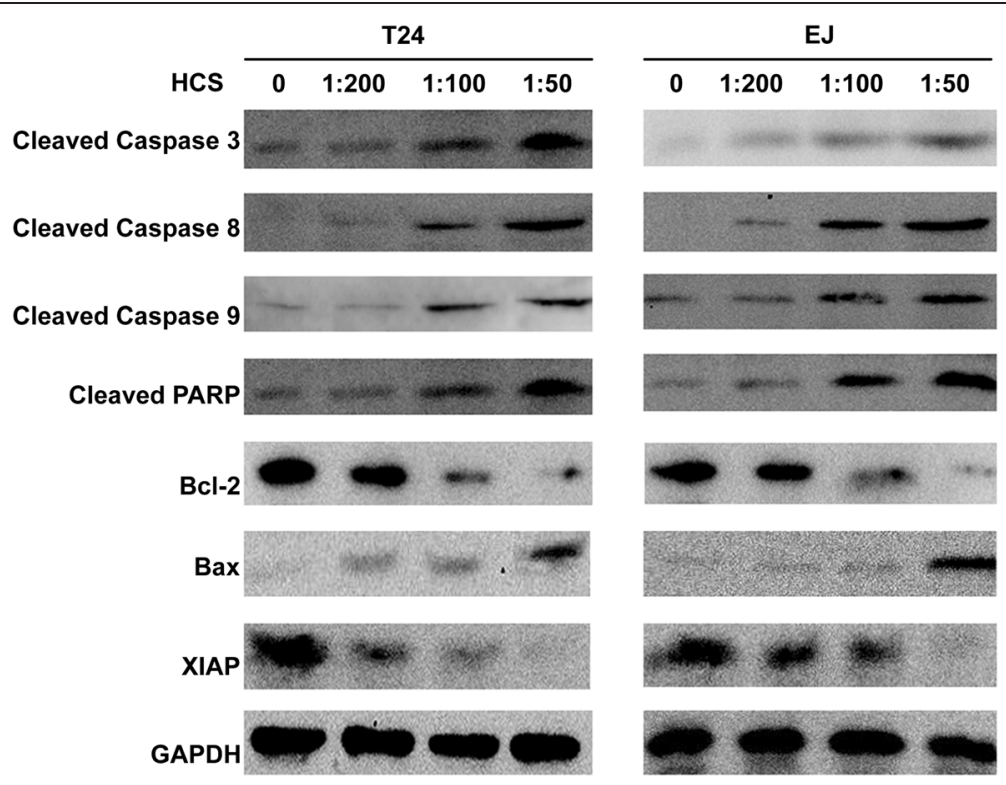

Figure 2 Effect of HCS on the levels of apoptosis-related molecules in T24 and EJ cell lines. The active (cleaved) forms of Caspase 3,8,9 and cleaved PARP were increased in a dose-dependent manner in HCS-treated T24 and EJ cells. the expression level of Bax was also elevated, whereas, the anti-apoptosis proteins such as BCl-2 and XIAP were down regulated after HCS treatment.

\section{HCS increases the expression of Fas, Fasl, TNF- $a$}

To explore the underlying molecular signaling pathways by which HCS exerts antitumor effects, we examined the expression of Fas/Fasl and TNF- $\alpha$ /TNFR1 by PCR, western blot immunohistochemistry and ELISA. As is shown in Figure 3A, B, the mRNA levels of Fas, Fasl,
TNF- $\alpha$ were increased in a dose-dependent manner in the two cell lines after HCS treatment. Furthermore, Western blotting results indicated that HCS treatment also increased the protein levels of Fas and Fasl, and ELISA results showed HCS could stimulate cells secrete TNF- $\alpha$ (Figure 3C, D). On the other hand, the expression

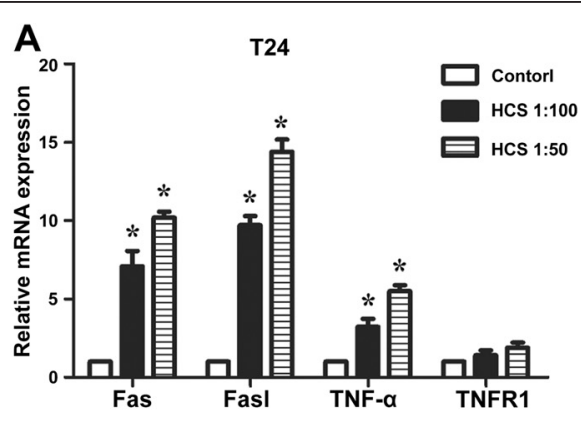

B

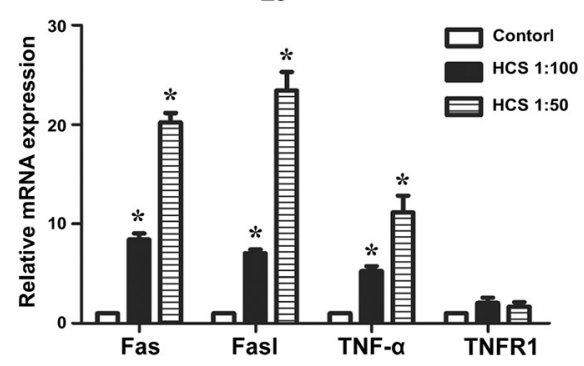

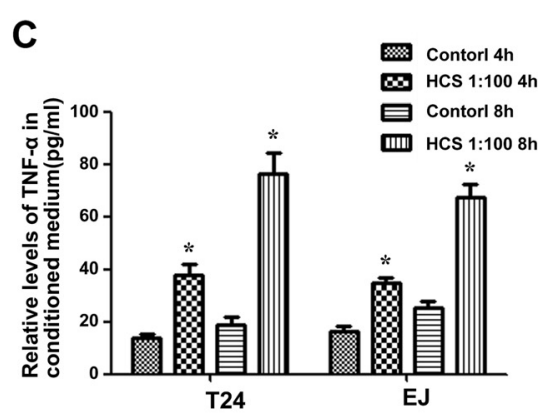

D

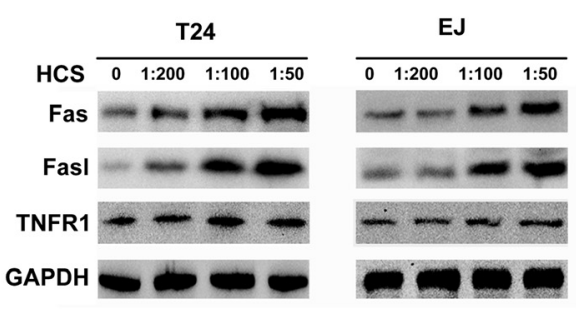

Figure 3 HCS treatment upregulated the expression of Fas, Fasl and TNF-a. The mRNA levels of Fas, Fasl, TNF-a and TNFR1 in T24 (A) and EJ (B) cells after HCS treatment. (C) Quantification of TNF-a in conditioned cell supernatant using ELISA. (D) The protein levels of Fas, Fasl and TNFR1 in T24 and EJ cells after HCS treatment were detected by western bolt. * $P<0.05$ vs control group. 
of TNFR1 in both mRNA and protein levels had no changes after HCS treatment as shown in Figures 3 and 4.

\section{HCS induced cell death is mediated by Fasl/Fas and TNF- $a$ /TNFR1 pathway}

To determine whether Fas/Fasl and TNF- $\alpha$ /TNFR1 expression is critical for the effect of HCS on bladder cancer cells, we knocked down TNF-a, TNFR1, Fas by siRNA or inhibited binding of FasL to its receptor by a blocking antibody (ZB4) prior to the addition of HCS, and then detected the cell viability. First, we confirmed that the siRNA effectively reduced the TNF-a, TNFR1, Fas mRNA level in T24 and EJ cells (Figure 5D, E). Then we found that TNF-a, TNFR1, Fas knockdown and the use of ZB4 significantly decreased the effect of HCS on cell proliferation compared with the control groups (Figure 5F, G). Furthermore, the effect of HCS on cell proliferation was more decreased by both of TNF-a/ Fas siRNA and TNF-a siRNA/ZB4 treatment, which implicated both Fas/Fasl and TNF- $\alpha / T N F R 1$ as mediators of HCS-induced cell death.

\section{Activation of NF-KB pathway against HCS induce cell death}

We also detected the activation of NF-kB pathway in T24 and RT-4 cells treated with HCS. Western blotting results showed HCS treatment decreased the expression of $i \kappa B-\alpha$ and increased the expression of p-p65 in a dose-dependent manner, which indicated NF- $\mathrm{kB}$ pathway was activated by HCS (Figure $5 \mathrm{~A}$ ). To investigate whether NF- $\mathrm{KB}$ pathway got involved in HCS induced cell death, we tried inhibiting NF- $\mathrm{kB}$ activity with PDTC before HCS treatment, then we compared the cell viability with that of HCS treated alone. Interestingly, when we pretreated T24 and RT-4 cells with NF-kB inhibitor PDTC for $1 \mathrm{~h}$ before HCS treatment, the effect of HCS on the cell proliferation was strongly enhanced (Figure 5B, C). The results demonstrated that NF-kB pathway could attenuated the effect of HCS on bladder cancer cell growth.

\section{HCS inhibits human bladder tumor xenograft growth in athymic nude mice}

We further investigated the effect of HCS on a transplanted tumor growth produced by T24 cells. Only two mice did not survive at the end of the experiment. As shown in Figure 6, the growth of T24 tumor xenografts was inhibited significantly following the injection of HCS at the dose levels of 1:2 and 1:1. The average bodyweight of the control group drops much more than the treatment groups $(\mathrm{P}<0.05)$, and it's speculated that the rapidly lose of bodyweight of the control mice may
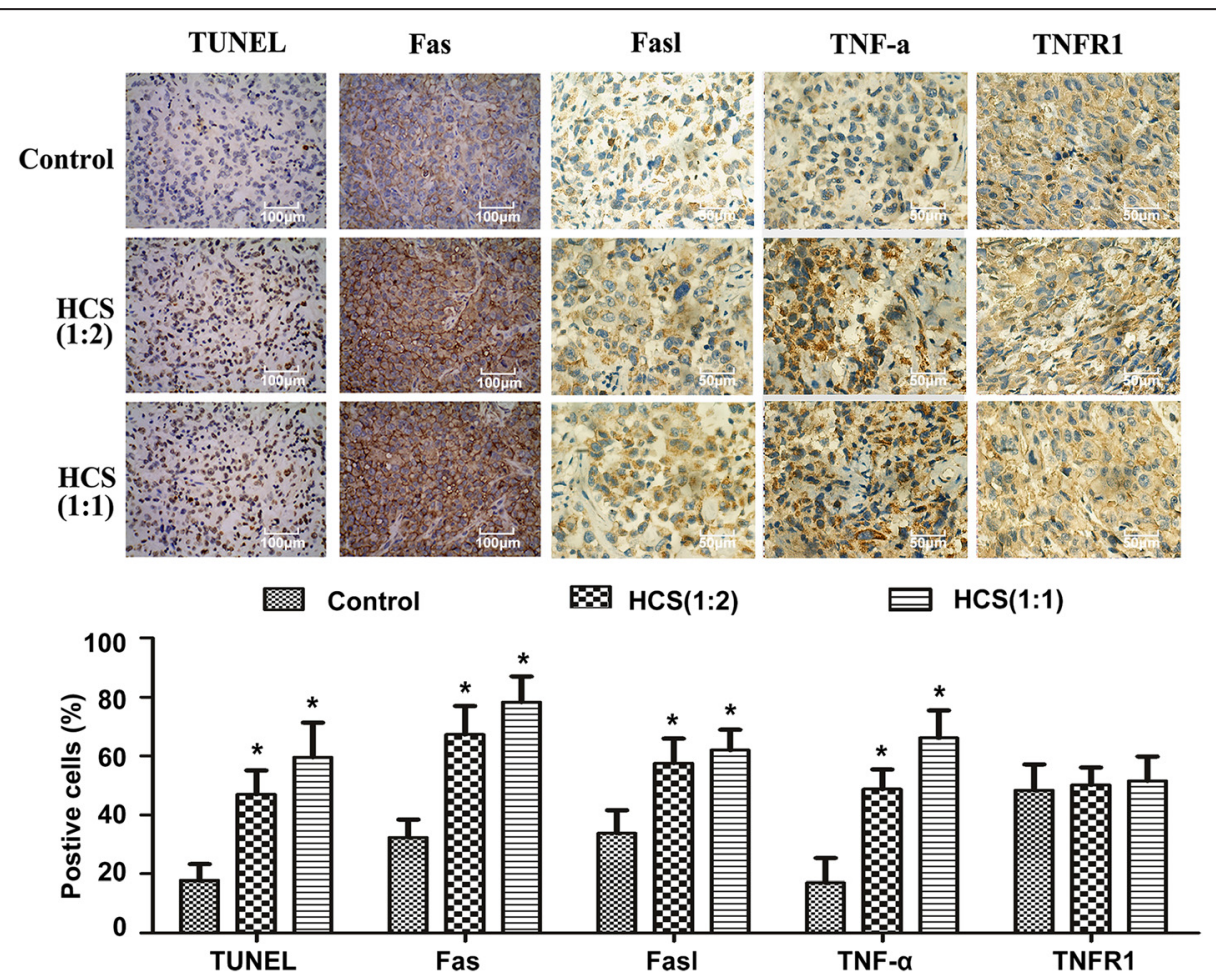

Figure 4 HCS induced cell apoptosis and the expression of Fas, Fasl and TNF-a in vivo. Representative results of the TUNEL staining of tumor sections and immunohistochemical analysis of Fas, Fasl, TNF-a and TNFR1 expression in control and HCS-treated T24 cell-transplanted mice. Columns are expressed as mean \pm SD of five samples of each group. ${ }^{*}, P<0.05$ compared to the control group. 


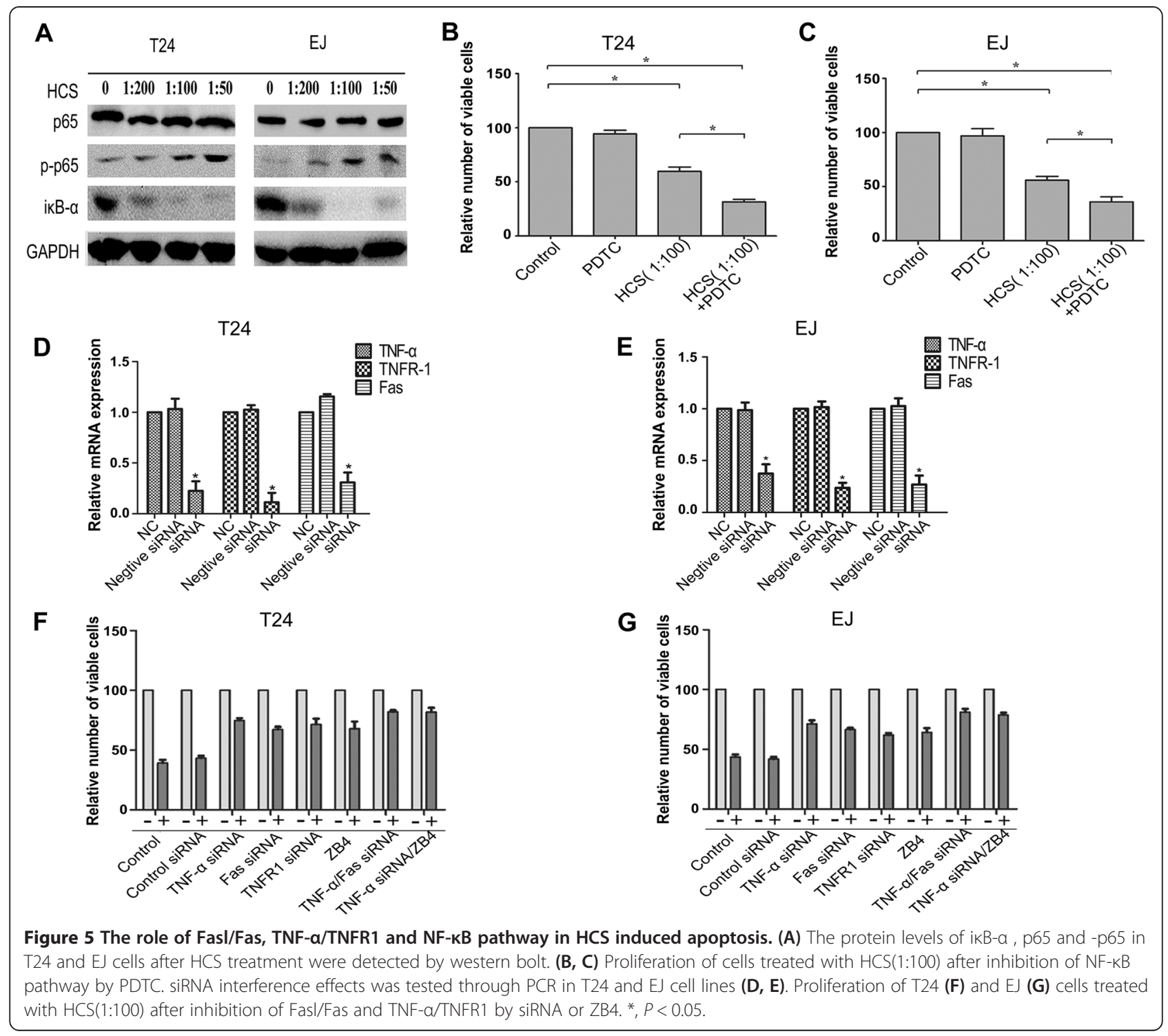

partially due to the progress of xenograft tumor.Besides, the average tumor masses in the control mice were nearly 2 fold $(\mathrm{P}<0.05)$ greater than that of HCS-treated mice(1:1), while there is no significant difference between the two HCS treatment group $(P>0.05)$. The apoptosis of tumor cells was evaluated using TUNEL stain. The apoptotic cells were more prominent in the HCS-treated tumors than that of the control tumors as is seen in Figure 4. Then We detected the expression of Fas, Fasl, TNF- $\alpha$ and TNFR1 by IHC , and the increases in protein level of Fas, Fasl, TNF- $\alpha$ were also observed in the tumors of HCS-treated mice.

\section{Discussion}

In recent years, the search for alternative anticancer agents in traditional medicine has been a potential strategy $[16,17]$. In this study, we explored that HCS induces apoptosis not only in cell culture, but also in tumor cells in vivo, as demonstrated in bladder cancer xenograft model treated with non-toxic dilutions of HCS.

To reveal the effect of HCS on bladder cancer cell lines, we tested the effect of HCS on cell growth and apoptosis. As revealed by MTS assay, HCS possessed an inhibitory ability on cell viability in a dose-dependent manner, while the inhibition is less remarkable in less malignant RT-4 and SV-HUC-1 cells. This phenomenon indicated that HCS may have less damage on normal bladder tissue. Furthermore, the morphologic changes induced by HCS indicate that cells were undergoing apoptosis. With annexin V/PI staining, HCS treatment significantly increased the proportion of apoptotic cells, which is increased with the rise of the concentration. These results confirmed that HCS exhibited an evident apoptosis-inducing effect on bladder cancer cells. 
A

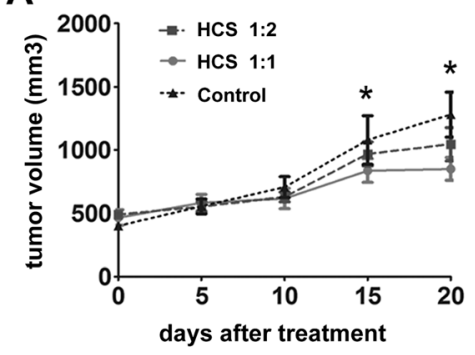

C

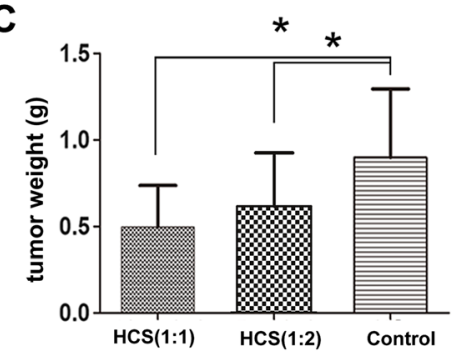

B
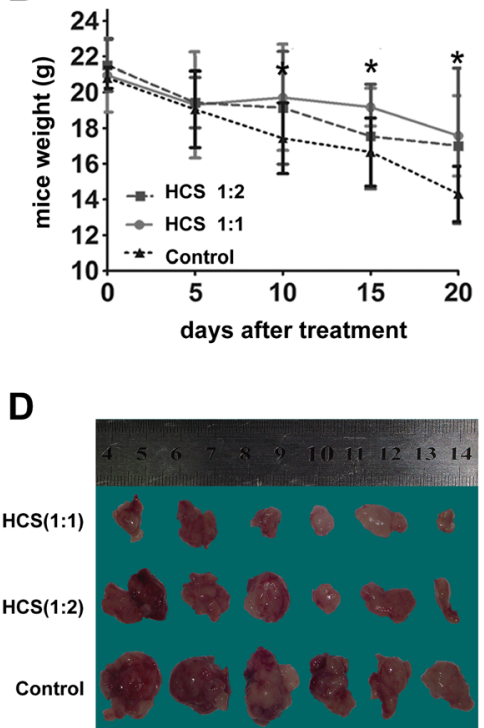

Figure $\mathbf{6}$ HCS inhibited tumor growth in vivo. Mean of tumor volume (A), body weight of mice (B) and tumor weight (C) measured at the indicated number of days after mice were treated with HCS. (D) Representative pictures of tumor in control and HCS-treated T24 cell-transplanted mice. ${ }^{*}, P<0.05$ compared to the control group.

Caspases, a family of cysteine-aspartic proteases, play an essential role in apoptosis. Caspases are first synthesized as inactive pro-Caspases and regulated at a posttranslational level. Activated Caspases eventually lead to apoptotic cell dismantling [18]. Poly (ADP-ribose) polymerase (PARP), a family of proteins involved in a variety of cellular processes including DNA repair and programmed cell death, is a downstream target of Caspase-3 $[19,20]$. Inhibitors of apoptosis proteins (IAPs) and Bcl-2 family proteins have also been shown to be important in the regulation of apoptosis $[21,22]$. We therefore tested the expression of Bax, Bcl-2, XIAP, cleaved PARP, cleaved Caspases 3, 8, 9, all are important members involved in apoptosis, through western blot after HCS treatment. The results confirmed that the apoptosis induced by HCS was related with the changes of these protein expression. So, what actually activated the Caspase cascade and initiated the apoptosis in bladder cancer cells treated with Huachansu? We tried to explore the result from both the intrinsic and extrinsic apoptosis pathway. The mitochondria-initiated intrinsic pathway, in which the release of cytochrome c from the mitochondrial matrix following loss of inner mitochondrial membrane integrity triggers formation of the apoptosome composed of Apaf-1, pro-Caspase-9, dATP, and cytochrome c [23]. The death receptorinitiated extrinsic pathway is another apoptosis process, in which death receptor ligation is followed by recruitment of adaptor molecules and activation of Caspase- 8 or
Caspase-10 [24,25]. We failed to detect the expression of cytochrome $\mathrm{c}$ by western blot for several times (data not shown), while the expression of Fas, Fasl, TNF- $\alpha$ were all elevated at both mRNA and protein level after HCS treatment. This results prompt us that the death receptor-initiated extrinsic pathway may contribute to the apoptosis induced by HCS in bladder cancer cells.

Fas is a member of the death receptor family, a subfamily of the tumor necrosis factor receptor superfamily. Binding to Fas by its physiological ligand, FasL, results in recruitment of adaptor molecules and activation of Caspase- 8 or Caspase-10, to form death inducing signaling complex (DISC), transducing a downstream signal cascade resulting in apoptosis [26,27]. TNF- $\alpha$ is a pleiotropic ligand of tumor necrosis factor receptor 1 and 2 (TNFR1 and TNFR2) that can signal both cell survival and cell death $[28,29]$. The death-inducing complex assembles following internalization of the TNFR1 and consists of TNFR1-associated death domain protein (TRADD) and receptor-interacting protein kinase 1 (RIPK1), which then recruit Fas-associated protein with death domain (FADD) and Caspase- 8 to generate the DISC [30]. In this study, to determine whether TNF-a/ TNFR1 and Fas/Fasl is required for HCS-induced cell death, we knocked down TNF-a, TNFR1, Fas by siRNA or inhibited binding of FasL to its receptor by a blocking antibody prior to the addition of HCS, then detected the cell viability. Importantly, downregulation of TNF-a, TNFR1, Fas or inhibition of Fas/Fasl interaction decreased the relative number of death cells induced by 
HCS, Figure 5 (F,G). Besides, TNF-a, TNFR1 and Fas knockdown with respective siRNA decreased the apoptosis rate induced by HCS in T24 cells, and the expression of cleaved caspase-3,-7,-8 and cleaved PARP also decreased as is shown in Additional file 1: Figure S1. Because of the above results we can conclude that HCS indeed affects bladder cancer cell growth which is mediated by TNF-a/TNFR1 and Fas/Fasl signaling pathway.

A major finding of this paper is that HCS not only reduced cell proliferation in cell culture but also inhibited tumor growth in vivo. We observed a significant increase in TUNEL-positive apoptotic cells in tumors from mice that were treated with HCS, and the protein level of Fas, Fasl, TNF- $\alpha$ in HCS-treated tumors were also up-regulated.

As we knew,the resistance of cells to TNF- $\alpha$ mediated apoptosisis attributed to TNF- $\alpha$ induced NF- $\kappa B$ activation [31], then we detected the activation of NF-kB pathway. As expected, NF- $\kappa B$ pathway was activated after HCS treatment. Then, we found inhibiton of NF- $k B$ by the inhibitor could enhance the effect of HCS-induced cell death. The results indicated that the treatment of HCS plus chemotherapeutic drugs which could inhibit NF- $\mathrm{kB}$ pathway in bladder cancer may have better curative effect compared to monotherapy.

\section{Conclusions}

Taken together, our study demonstrated that HCS induced-apoptosis in human bladder cancer was mediated by the activation Fas/Fasl and TNF- $\alpha /$ TNFR1. HCS may proved to be novel therapeutic strategy in the inhibition of carcinogenesis and progression of bladder cancer. Nevertheless, further studies are required to verify whether HCS could be used for an intravesical treatment.

\section{Additional file}

Additional file 1: TNF-a, TNFR1 and Fas siRNA transfection inhibited apoptosis of T24 cell induced by HCS. T24 cells are treated with TNF-a, TNFR1 and Fas siRNA for $48 \mathrm{~h}$ before HCS(1:100) treatment, And the apoptotic rate was detected by flow cytometry, the expression of cleaved PARP, XIAP and cleaved caspase-3,-7,-8 was detected by western blot. Additional file 1: Figure S1 (A,B) TNF-a, TNFR1 and Fas knock down decreased the apoptosis rates of HCS induced apoptosis in T24 cell, Columns are expressed as mean \pm SD of 3 independent experiments. ${ }^{*}, p<0.05$ for HCS vs. control, \#, p $<0.05$ for positive siRNA vs. negative siRNA and control + HCS. HCS, huachansu (1:100). (C) The expression of cleaved caspase-3,-7,-8 and cleaved PARP was decreased whereas the expression of XIAP elevated compared to the negtive control after respective siRNA transfection. Lane 1, 2, 3, 4, 5, 6 is consistent with the group order of that in Figure S1 B.

\section{Abbreviations}

HCS: Huachansu; TNF- a: Tumor necrosis factor- a; TNFR1: Tumor necrosis factor receptor 1; NF-kB: Nuclear factor kappa B; IAPs: Inhibitors of apoptosis proteins.

\section{Competing interests}

The authors declare that they have no competing interests.

\section{Authors' contributions}

TY, RS, LC performed the experiments. KT and GY analyzed the data. YT, Y, WH Contributed reagents/materials/analysis tools. $K C$ and $H X$ wrote the manuscript. XS and ZY conceived and designed the experiments. All authors read and approved the final manuscript.

\section{Acknowledgements}

This study was supported by the National Natural Science Foundation of China (31072238, 31172441, 31372562 and 81170650), and National Major Scientific and Technological Special Project for Significant New Drugs Development (2012ZX09303018).

\section{Author details}

'Department and Institute of Urology, Tongji Hospital, Tongji Medical College, Huazhong University of Science and Technology, Wuhan 430030, China. ${ }^{2}$ Department of Urology, Central Hospital of Wuhan, Wuhan 430014, China.

Received: 25 October 2014 Accepted: 10 February 2015

Published online: 25 February 2015

\section{References}

1. Bryan RT. Bladder cancer and cancer stem cells: basic science and implications for therapy. Sci World J. 2011;11:1187-94.

2. Arentsen HC, Hendricksen K, Oosterwijk E, Witjes JA. Experimental rat bladder urothelial cell carcinoma models. World J Urol. 2009;27:313-7.

3. Shen Z, Shen T, Wientjes MG, O'Donnell MA, Au JL. Intravesical treatments of bladder cancer: review. Pharm Res. 2008;25:1500-10.

4. Schenk-Braat EA, Bangma CH. Immunotherapy for superficial bladder cancer. Cancer Immunol Immun Cll. 2005;54:414-23.

5. Gartrell BA, Sonpavde G. Emerging drugs for urothelial carcinoma. Expert Opin Emerging Drugs. 2013;18:477-94.

6. Orang-Ojong BB, Munyangaju JE, Wei MS, Lin M, Wei FG, Foukunang C, et al. Impact of natural resources and research on cancer treatment and prevention: A perspective from Cameroon. Mol Clin Oncol. 2013;1:610-20.

7. Basmadjian C, Zhao Q, Bentouhami E, Djehal A, Nebigil CG, Johnson RA, et al. Cancer wars: natural products strike back. Frontiers Chem. 2014;2:20.

8. Qi F, Li A, Inagaki Y, Kokudo N, Tamura S, Nakata M, et al. Antitumor activity of extracts and compounds from the skin of the toad Bufo bufo gargarizans Cantor. Int Immunopharmacol. 2011;11:342-9.

9. Qi F, Inagaki Y, Gao B, Cui X, Xu H, Kokudo N, et al. Bufalin and cinobufagin induce apoptosis of human hepatocellular carcinoma cells via Fas- and mitochondria-mediated pathways. Cancer Sci. 2011;102:951-8.

10. Meng Z, Yang P, Shen Y, Bei W, Zhang Y, Ge Y, et al. Pilot study of huachansu in patients with hepatocellular carcinoma, nonsmall-cell lung cancer, or pancreatic cancer. Cancer. 2009;115:5309-18.

11. Ye M, Guo H, Guo H, Han J, Guo D. Simultaneous determination of cytotoxic bufadienolides in the Chinese medicine ChanSu by high-performance liquid chromatography coupled with photodiode array and mass spectrometry detections. J Chromatogr B Anal Technol Biomed Life Sci. 2006;838:86-95.

12. Watabe M, Ito K, Masuda Y, Nakajo S, Nakaya K. Activation of AP-1 is required for bufalin-induced apoptosis in human leukemia U937 cells. Oncogene. 1998;16:779-87.

13. Li D, Qu X, Hou K, Zhang Y, Dong Q, Teng Y, et al. PI3K/Akt is involved in bufalin-induced apoptosis in gastric cancer cells. Anti-Cancer Drugs. 2009;20:59-64.

14. Yu CH, Kan SF, Pu HF, Jea Chien E, Wang PS. Apoptotic signaling in bufalin- and cinobufagin-treated androgen-dependent and -independent human prostate cancer cells. Cancer Sci. 2008;99:2467-76.

15. Reis LO, Ferreira U, Billis A, Cagnon VH, Favaro WJ. Anti-angiogenic effects of the superantigen staphylococcal enterotoxin B and bacillus Calmette-Guerin immunotherapy for nonmuscle invasive bladder cancer. J Urol. 2012;187:438-45.

16. To KK, Au-Yeung SC, Ho YP. Differential nephrotoxicity of cisplatin and a novel series of traditional Chinese medicine-platinum anticancer agents correlates with their chemical reactivity towards sulfur-containing nucleophiles. Anti-Cancer Drugs. 2006;17:673-83. 
17. Mehta RG, Murillo G, Naithani R, Peng X. Cancer chemoprevention by natural products: how far have we come? Pharm Res. 2010;27:950-61.

18. Nicholson DW. Caspase structure, proteolytic substrates, and function during apoptotic cell death. Cell Death Differ. 1999;6:1028-42.

19. Burkle A, Brabeck C, Diefenbach J, Beneke S. The emerging role of poly (ADP-ribose) polymerase-1 in longevity. Int J Biochem Cell Biol. 2005;37:1043-53.

20. Yu SW, Andrabi SA, Wang H, Kim NS, Poirier GG, Dawson TM, et al. Apoptosis-inducing factor mediates poly(ADP-ribose) (PAR) polymer-induced cell death. Proc Natl Acad Sci U S A. 2006;103:18314-9.

21. Hunter AM, LaCasse EC, Korneluk RG. The inhibitors of apoptosis (IAPs) as cancer targets. Apoptosis Int J Program Cell Death. 2007;12:1543-68.

22. Heath-Engel HM, Chang NC, Shore GC. The endoplasmic reticulum in apoptosis and autophagy: role of the BCL-2 protein family. Oncogene. 2008;27:6419-33.

23. Huang X, Lu Z, Lv Z, Yu T, Yang P, Shen Y, et al. The Fas/Fas ligand death receptor pathway contributes to phenylalanine-induced apoptosis in cortical neurons. PLOS ONE. 2013;8:e71553.

24. Ashkenazi A. Targeting death and decoy receptors of the tumour-necrosis factor superfamily. Nat Rev Cancer. 2002;2:420-30.

25. Shimada K, Nakamura M, Ishida E, Kishi M, Yonehara S, Konishi N. c-Jun $\mathrm{NH} 2$-terminal kinase-dependent Fas activation contributes to etoposideinduced apoptosis in p53-mutated prostate cancer cells. Prostate. 2003:55:265-80.

26. Lavrik IN, Krammer PH. Regulation of CD95/Fas signaling at the DISC. Cell Death Differ. 2012;19:36-41

27. Kober AM, Legewie S, Pforr C, Fricker N, Eils R, Krammer PH, et al. Caspase-8 activity has an essential role in CD95/Fas-mediated MAPK activation. Cell Death Dis. 2011;2:e212.

28. Aggarwal BB. Signalling pathways of the TNF superfamily: a double-edged sword. Nat Rev Immunol. 2003;3:745-56.

29. Bhardwaj A, Aggarwal BB. Receptor-mediated choreography of life and death. J Clin Immunol. 2003;23:317-32.

30. Micheau O, Tschopp J. Induction of TNF receptor I-mediated apoptosis via two sequential signaling complexes. Cell. 2003;114:181-90.

31. Beg AA, Baltimore D. An essential role for NF-kappaB in preventing TNF-alpha-induced cell death. Science. 1996;274:782-4.

\section{Submit your next manuscript to BioMed Central and take full advantage of:}

- Convenient online submission

- Thorough peer review

- No space constraints or color figure charges

- Immediate publication on acceptance

- Inclusion in PubMed, CAS, Scopus and Google Scholar

- Research which is freely available for redistribution 